\title{
Effect of Shaddock Citrus Fruit Juice on the Lipid Profile, Fasting Blood Glucose Level and Kidney Function of Male Wistar Albino Rats
}

\author{
${ }^{1 *}$ Nwaka, Andrew C., ${ }^{2}$ Okeyikam, Colman C., ${ }^{3}$ Bayim,Peter-Robins B. and \\ ${ }^{4}$ Okafor, Irene N. \\ 1,2,4 Department of Biochemistry, Anambra State University, Uli, Nigeria. \\ ${ }^{3}$ Department of Chemical Sciences, Cross River State University of Technology, Calabar, Nigeria.
}

\begin{abstract}
The effect of shaddock citrus fruit juice on the lipid profile, fasting blood glucose level and some kidney function markers were evaluated in 16 male Wistar albino rats. The rats were procured from Faculty of Veterinary Medicine, Animal House, University of Nigeria, Nsukka and were of average age of 12 weeks, weighing 130 - 170grams. The rats were acclimatized for 7 days and randomly separated into 2 groups of eight rats each, kept in 2 different standard cages. The groups were as follows: A - rats fed with rat feed (Control), $B$ - rats fed with rat feed and shaddock citrus fruit juice. At the end of 28 days feeding experiment, the rats were bled and blood collected were used for lipid profile, fasting blood glucose level and kidney function assays using standard biochemical methods. The results of the study indicated a decrease in the blood glucose level of rats supplemented with shaddock citrus fruit juice (group B) when compared with those of the control (group $A)$. Result of the study also showed significant reduction $(p<0.05)$ in mean total cholesterol, low density lipoprotein $(L D L)$ and triglyceride concentrations of rats supplemented with shaddock citrus fruit juice when compared with those of the control. Moreover, the result revealed no significant difference $(p>0.05)$ in mean urea and creatinine concentrations of rats supplemented with shaddock citrus fruit juice when compared with those of the control. The result of this study suggests that shaddock citrus fruit juice was not toxic to the kidney and could assist in reducing the blood glucose level and protect against cardiovascular diseases and related complications.
\end{abstract}

Key Words: Shaddock citrus fruits juice, kidney function markers, blood glucose level, lipid profile and Wistar rats.

\section{Introduction}

The plant shaddock citrus fruit (Citrus maxima) or pomelo are native of Southeastern United States and they are also widely cultivated in some regions of Africa (Oyedepo, 2012). Shaddock is a hybrid of grape (Citrus vinifera) and orange (Citrus sinensis) with a smooth yellowish skin and may either be seeded or seedless. In some cases, through selection and breeding, shaddock has been crossed with tangerine (Citrus reticulate) to obtain a variant with juicy, thick skinned, easy to peel and tangelo (Arih, 2006). The pulp of shaddock citrus fruit is stated to possess appetizer, antitoxic, cardiac stimulant and stomach tonic properties, as reported in ancient and medieval literature (Arias and Roman - Laca, 2005). The major flavonones of shaddock citrus fruits are neohesperidin and narigin, which are higher, in the seed than in unripe fruit, (Chung et al., 2000), and its extract possessed antioxidant activity (Lim et al., 2006). Kidney function tests/markers measure various chemicals in the blood made by the kidney. An abnormal result indicates a problem with the liver, and may help to identify the cause. Further tests may be needed to clarify the cause of the kidney problems. Some of the kidney function markers include urea and creatinine (Cheesbrough, 2005). While lipid profile is a panel of blood tests that serves as an initial broad medical screening tool for abnormalities in lipid, such as cholesterol and triglycerides. Lipid profile include low density lipoprotein (LDL), high density lipoproteins (HDL) triglyceride and total cholesterol (Sidlin and Naugler, 2012).

The term blood glucose level or blood sugar concentration is the amount of glucose (sugar) present in the blood of a human or animal. The body naturally tightly regulates blood glucose levels as a part of metabolic homeostasis (Boiley et al., 2006). While the Wistar rat is an outbreed albino rat. These stocks were developed at the Wistar Institute in 1906 for use in biological and medicinal research and is notably the first rat developed to serve as a model organism at a time when laboratories primarily used the common house mouse. More than half of all laboratory rat strains descended from the original colony established by physiologist Henry Donaldson (Krinke, 2000). The Wistar rat is currently one of the most popular rats used for laboratory research. It is characterized by its wide head, long ears, and having a tail length that is always less than its body length.

Since shaddock citrus fruit has a lot of nutritive and medicinal potentials, the need to unravel its daily ingestion effect on the kidney function, blood glucose level and lipid profile of male Wistar albino rats was what this study aimed at. 


\section{Materials and Methods}

Shaddock: The shaddock citrus fruit was purchased from the local market at Ojoto, Idemili South Local Government Area, Anambra State. The shaddock citrus fruit juice was extracted by peeling off the back of the shaddock fruit and pounded with a pestle inside a mortar, after which the cheese cloth was used to sieve the filtrate (juice) out of the residue.

Chemicals: All the chemicals used in the study were of analytical grades.

Animals Used: Mature male Wistar Albino rats of average age of twelve (12) weeks weighing 130 - 170grams were procured from Faculty of Veterinary Medicine Laboratory Animal House, University of Nigeria, Nsukka Campus. The rats were acclimatized for seven (7) days before the commencement of the study. The rats were all supplied with normal standard pelleted growers mash of Vital feed and water ad libitum.

Rat Feed: The normal rat feed used in the study was pelleted growers mash of Vital ${ }^{\circledR}$ feed, produced by Grand Cereal and Oil Mills Jos, Nigeria.

\section{Experimental Design}

Sixteen (16) mature male Wistar rats weighing 130 - 170grams were used in the study. The rats were separated into two (2) groups of eight (8) rats each and kept in two (2) different standard aluminum cages as follows: group A - Wistar rats fed on rats feed only and group B - Wistar rats fed on rats feed and shaddock citrus fruit juice. All the protocols as approved by Institutional Animal Ethics Committee (IAEC) were observed in this study. The study lasted for 28days, while at the end of the 28days feeding experiment, the rats were bled from the retro-bulbar plexus of the medial cantus of the eyes. The blood samples were collected into plain sample bottles. The blood samples collected in the plain tubes without Ethylene Diamine Tetraacetic acid (EDTA) were allowed to stand for 30 minutes and clotted. The clotted blood was then centrifuged at the speed of 30,000 revolution per minutes for 10 minutes. The serum was extracted and used for kidney function markers and lipid profile assays using standard biochemical methods, while blood glucose level was determined using full blood that has not been treated.

Administration of shaddock citrus fruit juice: shaddock citrus fruit juice was given to the rats by administering $12 \mathrm{ml} / \mathrm{kg}$ body weight of the juice orally to the rats each morning before being fed for the day.

The fasting blood glucose level was done using prestige ${ }^{\circledR}$ smart system (Home Diagnostics Inc. USA) which was based on glucose oxidase method as reported by ADA (2003). The rats were fasted for 12hours before blood sample was collected from them for fasting blood glucose level test. Lipid profile was done using colorimeric method as reported by Allain et al.(1974) and Albert et al.(1978). Kidney function test was done using modified method as reported by Searcy et al. (1967).

\section{Statistical Analysis}

The data collected from the variables were expressed in mean and standard deviation. The data collected from the variables were subjected to student $\mathrm{t}$-test using the statistical package for social science (SPSS) version 17. Also student t-test was used in comparing the mean differences in variable amongst the two groups involved in this study. The acceptable level of significance was $p<0.05$.

\section{Result}

Table 1: Fasting Blood Glucose level of Rats used in the study

\begin{tabular}{|l|l|}
\hline Group & Blood glucose level $(\mathrm{mg} / \mathrm{dl})$ \\
\hline A & $81.00 \pm 8.83$ \\
\hline B & $78.75 \pm 5.74$ \\
\hline
\end{tabular}

The result of fasting blood glucose level (Table 1) showed that there was a decrease in blood glucose level of rats supplemented with shaddock citrus fruit juice (group B) when compared to those of the control (Group A).

Table 2: Serum lipid profile concentration of Rats used in the study

\begin{tabular}{|l|l|l|l|l|}
\hline Group & Total Cholesterol $(\mathrm{mg} / \mathrm{dl})$ & HDL $(\mathrm{mg} / \mathrm{dl})$ & LDL $(\mathrm{mg} / \mathrm{dl})$ & Triglyceride $(\mathrm{mg} / \mathrm{dl})$ \\
\hline A & $82.39 \pm 5.75$ & $32.39 \pm 1.67$ & $37.72 \pm 6.48$ & $60.49 \pm 20.12$ \\
\hline B & $69.87 \pm 2.07$ & $27.83 \pm 1.46$ & $28.40 \pm 2.90$ & $37.76 \pm 11.14$ \\
\hline
\end{tabular}

Table 2 revealed that there was significant reduction $(\mathrm{P}<0.05)$ in mean total cholesterol, low density lipoprotein (LDL) and triglyceride concentrations of rats supplemented with shaddock citrus fruit juice (group B) when compared to those of the control (Group A). The result also indicated that there was no significant difference $(\mathrm{P}>0.05)$ in the mean serum high density lipoprotein (HDL) concentration of rats supplemented with shaddock citrus fruit juice (group B) when compared to those of the control (group A). 
Table 3: Correlation Existing Between Total Cholesterol, HDL, LDL and Triglyceride in the study

\begin{tabular}{|l|l|l|l|l|l|}
\hline & & $\begin{array}{l}\text { Total Cholesterol } \\
(\mathrm{mg} / \mathrm{dl})\end{array}$ & HDL(mg/dl) & LDL(mg/dl) & Triglyceride(mg/dl) \\
\hline $\begin{array}{l}\text { Total } \\
\text { Cholesterol }\end{array}$ & $\begin{array}{l}\text { Pearson } \\
\text { Correlation }\end{array}$ & 1 & 0.34 & $0.74^{*}$ & 0.13 \\
\hline$(\mathrm{mg} / \mathrm{dl})$ & Sign (2 tailed) & & 0.21 & 0.00 & 0.64 \\
\hline & $\mathrm{N}$ & 16 & 16 & 16 & 16 \\
\hline
\end{tabular}

Table 3 above indicated that there was a very strong positive correlation between LDL and total cholesterol, which suggests that as the total cholesterol increases ,LDL also increases. There was also a little positive correlation between triglyceride, HDL and total cholesterol but was not statistically significant.

Table 4: Concentrations of some kidney Function Markers of Rats used in the study

\begin{tabular}{|l|l|l|}
\hline Group & Urea $(\mathrm{mg} . \mathrm{dl})$ & Creatinine $(\mathrm{mg} / \mathrm{dl})$ \\
\hline A & $30.27 \pm 1.91$ & $0.62 \pm 0.12$ \\
\hline B & $28.49 \pm 4.05$ & $0.65 \pm 0.02$ \\
\hline
\end{tabular}

The result in Table 4 indicated that there was no significant difference $(\mathrm{P}>0.05)$ in mean urea and creatinine concentrations of rats supplemented with shaddock citrus fruit juice (group B) when compared to those of the control (group A).

\section{Discussion}

The pulp of shaddock citrus fruit has been reported to posses some nutritive and medicinal potentials (Arias and Ramon-Laca, 2005). In this study, the effect of shaddock citrus fruit juice on the kidney, blood glucose level and lipid profile parameters of male Wistar albino rats were evaluated.

The result of fasting blood glucose level showed that there was reduction in blood glucose level of rats supplemented with shaddock citrus fruit juice (group B) when compared to those of the control (group A).The result of this study suggests that shaddock citrus fruit juice could assist in reducing the blood glucose level. This work is in agreement with Betteridge (1997) who also discovered that shaddock citrus fruit juice decreases the blood glucose level. This suggests that shaddock citrus fruit juice could be a good supplement for diabetics, since it could assist in stabilizing their blood glucose level.

Furthermore, the result of lipid profile in Table 2 revealed that there was significant decrease $(\mathrm{P}<0.05)$ in total cholesterol level of rats supplemented with shaddock citrus fruit juice (group B) when compared with those fed with normal rat feed only, group A (control). This observation is in agreement with the work of Oyedepo and Babatunde (2013) who also discovered that shaddock citrus fruit juice significantly reduced the total cholesterol level of streptozotocin induced diabetic rats. Moreso, the result in Table 2 indicated significant decrease $(\mathrm{p}<0.05)$ in low density lipoprotein $(\mathrm{LDL})$ and triglyceride levels of rats supplemented with shaddock citrus fruit juice (group B) when compared with those of the control (group B). This observations is also in agreement with the works of Oyedepo and Babatunde (2013) who also observed decrease in LDL and triglyceride levels of diabetic rats supplemented with shaddock citrus fruit juice. The results of this study therefore suggest that shaddock citrus fruit juice could protect against cardiovascular diseases and related complications, since according to Brown et al. (1993), reducing the serum lipid levels through dietary or drug therapy decreases the risk of cardiovascular disease and its related complications. Additionally the result of lipid profile correlation study (Table 3) showed a very strong positive correlation existing between total cholesterol level and LDL. This implied that as the total cholesterol level is increasing, the LDL concentration will also be increasing and vice versa.

Finally, the result of some kidney function markers assayed in the study (Table 4) revealed that there was no significant difference $(\mathrm{P}>0.05)$ in the urea and creatinine concentrations of rats supplemented with shaddock citrus fruit juice (group B) when compared with those of the control (group A). The result of this study suggests that shaddock citrus fruit juice was not toxic to the kidney of rats used in the study.

\section{Conclusion}

The result of this study suggests that shaddock citrus fruit juice daily ingestion at the dose of $12 \mathrm{ml} / \mathrm{kg}$ body weight was not toxic to the kidney, since all the kidney function parameters examined were still within the normal range. The result of the study also showed reduction in the blood glucose level of rats supplemented with shaddock citrus fruit juice when compared to those of the control. This suggests that shaddock citrus fruit juice could assist in reducing the blood glucose level and could also be a good supplement for diabetics since it could assist in stabilizing their blood glucose level. Moreover, the result of the lipid profile in this study indicated significant decrease $(\mathrm{P}<0.05)$ in total cholesterol, LDL and triglyceride of rats supplemented with shaddock 
citrus fruit juice (group B) when compared with those of the control. This suggests that shaddock citrus fruit juice could assist in protecting against cardiovascular disease and its related complications.

\section{References}

[1]. Alberts, J.J., Warnick, G.R. and Chung, M.C. (1978). Quantification of High Density Lipoprotein.Journal of Biochemistry, 13:926932.

[2]. Allain, C.C., Poon, L.S., Chan, C.S., Richmond, W. and Fu, P.C. (1974). Enzymatic Determination of Total Cholesterol. Journal of Clinical Chemistry, 20: 470-475.

[3]. Arias, B.A. and Ramon-Laca, L. (2005). Pharmacological properties of citrus and their ancient and medieval uses in the Mediterranean region. Journal of Ethaopharamacology 97: 8995.

[4]. Arih, A.C. (2006). Production and evaluation of skarkling wire from shaddock (Citrus maxima) fruit using palm wine yeast (Saccharomyces ceresiae) isolate. Proceedings of the $30^{\text {th }}$ Annual Conference of Nigeria Institute of Food Science and Technology, 30: 3839 .

[5]. Betteridge, J. (1997). Lipid Disorders in Diabetes Mellitus, Textbook of Diabetes. $2^{\text {nd }}$ Edition, Blackwell Science, London,Pp. 1-55.

[6]. Boily, F., Beaudoin, S. and Measures, L.N. (2006). Haematology and Serum Chemistry of the Harp and Hooded Seals during the Breeding Season in the Gulf of Saint Lawrence. Canada Journal of Wildlife Diseases, 42: 115-132.

[7]. Brown, G.B., Xue-Qiao, Z., Sacco, D.E. and Alberts, J.J. (1993). Selenium, Selenoproteins and Human Health.Journal of Public Health Nutrition, 4:593-599.

[8]. Cheesbrough, M. (2005). Clinical chemistry tests. Distruct laboratory practice in tropical countries $1^{\text {st }}$ edition Cambridge University Press, New York, Pp. 310360

[9]. Chung, S.K., Kim, S.H., Choi, Y.H., Song, E.Y. and Kim, S.H. (2000). Status of citrus fruit production and view of utilization in chejin. Journal of Food Industry Nutrition, 5: 4252.

[10]. Krinke, G.J. (2000). History, Stains and Models:The Laboratory Rat, Animal Science. $1^{\text {st }}$ Edition, Academic Press, New York, Pp. 3 16.

[11]. Lim, H.K., Yoo, E.S., Moon, J.Y., Jeon, Y.J. and Cho, S.K. (2006). Antioxidant Activity of Extracts from Dangyja fruit produced in Jeju Island. Journal of Food Science Biotechnology 15: 412316.

[12]. Oyedepo, A. and Babatunde, S.O. (2013). Effect of shaddock fruit juice on Glucose tolerance and lipid profile in Type II Diabetic Rats. Journal of Chemical Science Transactions 2: 1920

[13]. Oyedepo, T.A. (2012). Effect of Citrus maxima fruit juice in Alloxan induced diabetic wistar rats. Science Journal of Medicinal and Clinical Trial 2012: 125128

[14]. Searcy, R.L., Reardon, J.E. and Foreman, J.A. (1967). A New PhotometricMethod for Serum Urea Nitrogen Determination. American Journal of Medical Technology, 33:15-20.

[15]. Sidhu, D. and Naugler, C. (2012). Fasting Time and Lipid Levels in a Community. Journal of Medicinal Food,10: 1-4.

[16]. Wikipedia, ADA (American Diabetes Association) (2003). Total Prevalence of Diabetes and Pre- diabetes. Retrieved on 2006 -03-17. 